\title{
Who shot optical packet switching?
}

\author{
José Roberto de Almeida Amazonas*†, \\ Germán Santos-Boada, and Josep Solé-Pareta* \\ * Department of Computer Architecture, Technical University of Catalonia, Barcelona, Spain \\ $\dagger$ Escola Politécnica of the University of São Paulo, São Paulo, Brazil
}

\begin{abstract}
Looking at the volume of publications dealing with Optical Packet (OPS) and those dealing with Elastic Optical Networking (EON) in the period from 2000 to 2016, one clearly can observe that between 2004 and 2009 there is a boom of the OPS publications and then they start to diminish. At the same time, EON publications start to take-off (around 2011) and is steadily growing. EON is an emerging technology that may be considered as a killer technology in the sense that its performance, flexibility, associated cost, ease of deployment and marketing seem to be displacing OPS from the optical networking arena. The purpose of this paper is discuss if, effectively, EON is the killer technology of OPS. So then, besides review the EON technology, the paper analyzes its actual deployment possibilities.
\end{abstract}

\section{Keywords}

Elastic Optical Networks (EONs); Optical Burst Switching; Optical Packet Switching.

\section{INTRODUCTION}

When optical networks became an excellent solution for transmitting data based on the improvement of delay and data rate related to digital switching, two optical switching technologies, Optical Packet Switching (OPS) and Optical Burst Switching (OBS), have been the focus of the research activities for a long time. However, the unavailability of devices to implement these technologies has driven the appearance of a new technology based on flexi-grid spectrum, Elastic Optical Networks (EONs), and OPS/OBS had a significant decline in the number of scientific publications, as can be seen in Figure 1.

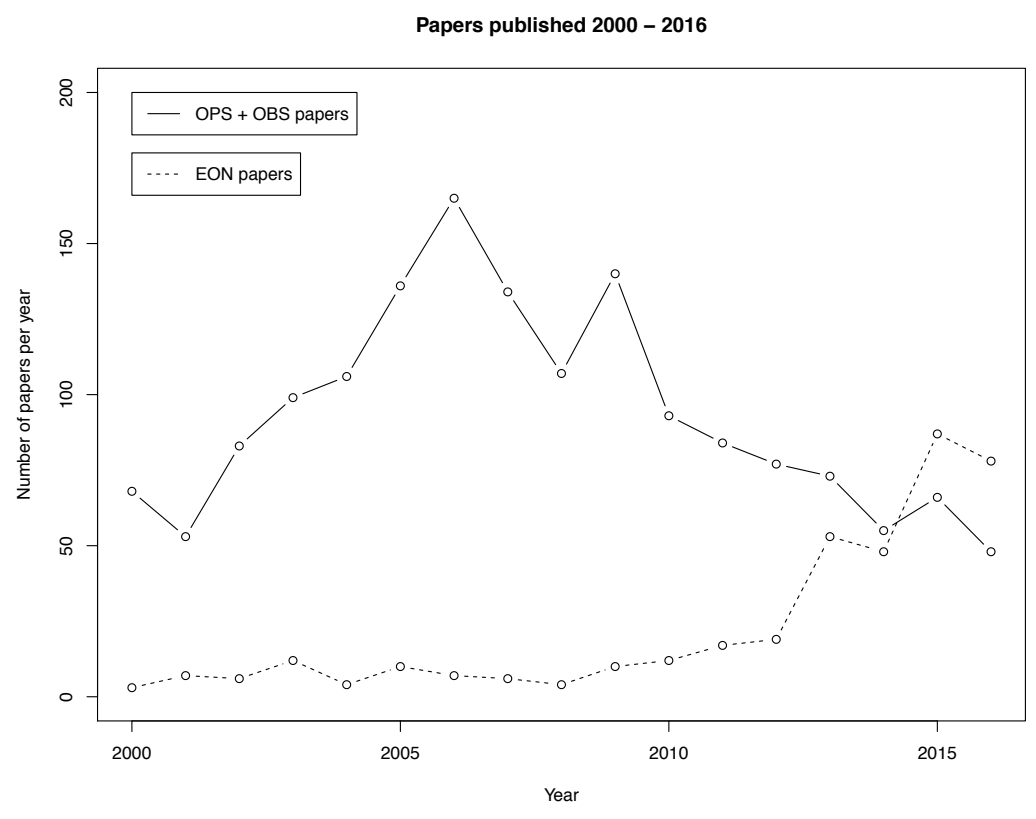

Fig. 1. OPS, OBS and EON published papers per year between 2000 and 2016.

It is clear that OPS/OBS hype occurred around 2004-2009 and then it started to decrease. On the other hand, the interest in the EON technology started around 2011 and is steadily growing. EON is an emerging technology that may be considered a killer in the sense that its performance, flexibility and associated cost seem to be displacing OPS/OBS from the optical networking technological arena. The main features of switching technology that are susceptible of being analyzed and the 
reason for the change of technology trend are: delay, data rate, resource optimization, complexity, performance, flexibility, associate cost, ease of deployment, interaction with upper network layers.

After this brief introduction, Section II makes a review of the EON technology, Section III analyzes its deployment possibilities and in Section IV the conclusions are presented.

\section{Characteristics AND DePloyment hindRANCES OF THE EON TECHNOLOGY}

In this section we present a timeline of the EON technology development emphasizing network performance, critical devices and economic constraints. This provides an overview that helps to infer the possibilities of the technology deployment in a foreseeable future.

a) 2009, [1] - The introduction of SLICE: The extended distance an optical signal can travel through multiple DWDM links and wavelength cross-connects (WXCs) without undergoing optical-electrical- optical (OEO) regeneration has made the optically routed transparent mesh networks feasible. The optically routed transparent mesh networking has a drawback with respect to the stranded bandwidth due to its rigid large granularity, and the efficient utilization of the deployed network capacity is one of the network operators' major concerns. Another important challenge of a network operator is to accommodate the super- wavelength data traffic for cutting edge enterprise and scientific customers in a cost-effective and scalable manner. Several approaches such as OPS, waveband switching, or optical virtual concatenation (OVC) have been proposed to meet the above mentioned demands offering sub- and super-wavelength traffic accommodation. However, their enabling technologies were still in the primary development stage, or lead to inefficiencies in bandwidth allocation, motivating the introduction of a novel spectrum efficient and scalable optical transport network architecture called spectrum-sliced elastic optical path network (SLICE). Since SLICE offers a coarser granularity than OPS, it was considered by the proposers as a middle-term alternative to the then immature OPS technology.

b) 2010, [2] - Distance-adaptive spectrum resource allocation: Another limitation of the optical networks is associated with worst case design in terms of transmission performance. In order to address this problem, a concept of a novel adaptation scheme in SLICE called distance-adaptive spectrum resource allocation was presented, in which the minimum necessary spectral resource is adaptively allocated according to the end-to-end physical condition of an optical path. Modulation format and optical filter width are used as parameters to determine the necessary spectral resources to be allocated for an optical path.

c) 2011, [3] - Dynamic Elastic and Scalable Photonic Infrastructures and Network Architectures ? DESPINA approach: The DESPINA approach foresaw to extend the capabilities of existing networks, rather than proposing a revolutionary alternative approach (unlike OBS/OPS), in order to obtain benefits in a similar manner to the benefits that reconfigurable and wavelength tuneable solutions (e.g. ROADMs and WSSs) offered in the past.

d) 2013, [4] - SLICE evolves to EON: The initially proposed SLICE architecture has evolved to become the Elastic Optical Network (EON) concept. The EON allows flexible allocation of appropriate-sized optical bandwidth, by means of contiguous concatenation of optical spectrum, to an end-to-end lightpath according to traffic demand. Two components are essential for EON architectures: bandwidth-variable transponders (BV-Ts) and bandwidth-variable wavelength cross-connects (BV-WXCs). The role of a BV-T is to adapt the client data signal to be sent to/received from the optical network with just enough frequency resources. Concurrently, BV-WXCs allow the creation of an optical routing path through the network by switching transmitted signals within their frequency bandwidth to appropriate switch output ports.

e) 2013, [5] - OFDM-based elastic optical network architecture: In the Orthogonal Frequency Division Multiplex OFDM-based elastic optical network architecture, multiple data rate sub-wavelength or super-wavelength paths are realized through flexible granular grooming and switching in the spectrum domain, using data-rate/bandwidth-variable transponders and bandwidth-variable WXCs. The data-rate/bandwidth-variable transponder allocates just enough spectrum (subcarriers) to accommodate sub-wavelength traffic, known as spectrum slicing. Several OFDM channels can be merged together into a superchannel, transporting a multiple of the capacity of an individual OFDM channel without spectrum guard band in between, as shown in Fig. 2(a). Every WXC node on the optical path allocates a cross-connection with the appropriate spectrum bandwidth to create an appropriate-sized end-to-end optical path. When the traffic increases, the transmitter can increase the transmission capacity, and every WXC on the route expands the switching window, thus enabling data-rate/bandwidth-variable optical paths. Fig. 2(b) shows the architecture of the OFDM-based elastic optical network.

f) 2013, [6] - The mixed-line-rate (MLR), bandwidth-variable (BV) and multi-flow (MF) models: The MLR model employs a few types of transponders, each with a different bit rate. This study examined 40, 100 and 400 Gbps transponders to suit a wide range of traffic demands. The BV model supports all types of traffic demands with a BV transponder. This study examined a transponder whose maximum bitrate was $400 \mathrm{~Gb} / \mathrm{s}$, and that assigned the fewest possible spectral resources to support traffic demands. The MF model uses a MF transponder, with 10 sub-transceivers, each of which has $40 \mathrm{~Gb} / \mathrm{s}$ capacity. The optical node scalability result depends not on the transponder cost, but on the number of transponders or the capacity of optical paths. Evaluation results showed that the MF model needs about 50\% fewer add/drop parts than the MLR and BV models. A cost evaluation showed that the advantage of resource sharing provided by the MF model also leads to a reduction in network cost. 


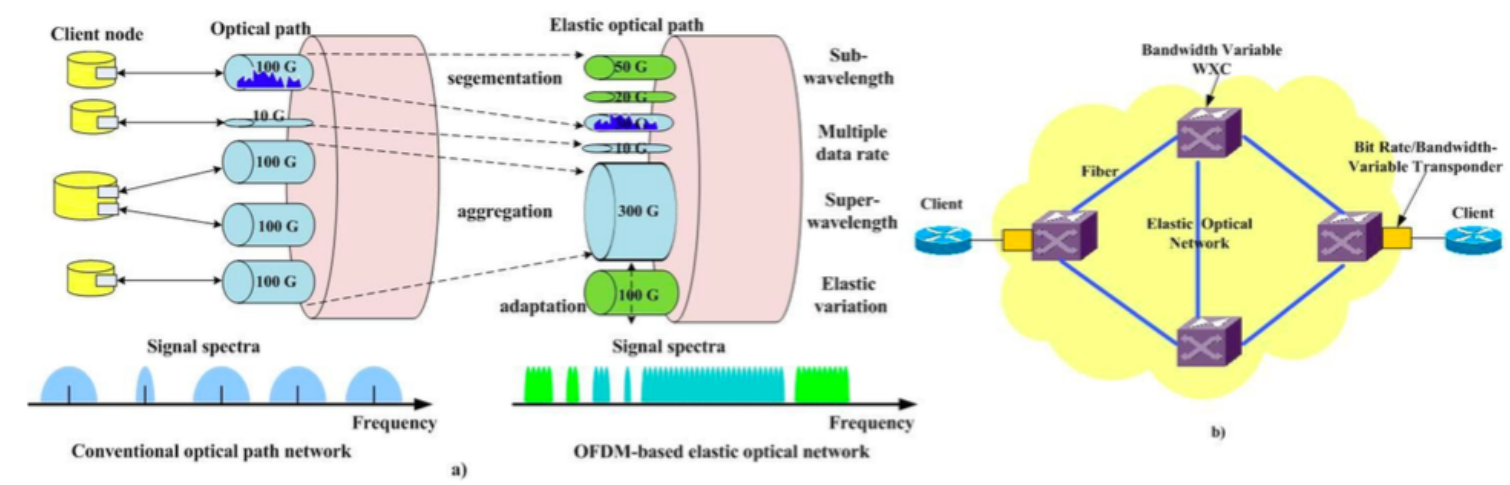

Fig. 2. Architecture of OFDM-based elastic optical network: a) Comparison between conventional and elastic optical paths, b) Architecture of elastic optical network - Source: [5].

g) 2014, [7] - Operators would like to migrate their infrastructure to use SBVTs if 50\% savings are possible: For a full mesh topology in almost every instance a $50 \%$ overall savings is possible when unit costs of the SBVT are higher than the cost of non-sliceable transponders. For an IP topology in almost every instance the 50\% overall savings are possible even when the unit cost of SBVTs is higher than the cost of non-sliceable transponders. Full mesh brings more benefits by using an SBVT because the traffic is distributed over all nodes, taking maximum advantage of the main characteristic of the SBVT transponders, to tune their bandwidth. On the other hand, in IP topology the traffic is routed through the network connecting the shortest path between nodes, which implies an increase of traffic per node. Higher traffic uses more capacity of the SBVT, losing part of its main advantage. IP topology is more realistic than full mesh because it reduces the number of IP ports. Also, in terms of total cost to deploy a network, IP topology is better for the operators.

h) 2014, [8] - Migration to EON will not be attractive before 2019: From the network operator perspective, it is important to know when this augmentation of capacity will represent a real solution for their networks, in other words, when the network capacity will be a problem. There are two possible cases in the EON model: 1) it is possible to activate the flexi-grid functionality in the WSSs by a software upgrade and 2) the deployed WSSs cannot implement flexi-grid capability, and they would need to be replaced. The reference network is the Spanish Transport network. The results show that a network based on flexi-grid could extend the lifetime of the network five years with respect to the legacy DWDM. Results also show that current DWDM capacity will not be exhausted until 2019. The pure argument of capacity improvement is not a short-term driver to deploy flexi-grid solutions. Even though in the next few years capacity in terms of spectrum is not yet an issue, and thus migrating to flexi- grid just because of the lack of spectrum makes it not urgent, a key driver to migrate to flexi-grid is the demand of high-speed channels and the availability of cost-effective 400 Gbps and 1 Tbps transponders.

i) 2015, [9]- New requirements need to be addressed in future high-capacity flexi-grid optical nodes: Next generation optical nodes will incorporate high levels of flexibility in different domains, such as switching, bandwidth, and transmission rate. Optical node scalability is another important requisite supported by a component's modularity to achieve a practical approach and enable future services to easily be added to the network functionality. Node resilience is also accounted for in EONs. Node failures due to faulty subsystems should be efficiently addressed and overcome, guaranteeing continuously rapid provision of services to network users. Node adaptability to new network applications is also preferred for these emerging optical nodes. Addressing the emerging requirements for flexibility, scalability, resilience, and adaptability, the Architecture on Demand (AoD)-based Flex-OXC nodes was proposed.

j) 2015, [10]-EON is a promising concept but its implementation remains some way off: One of the important challenges faced by the research community is to develop a new sliceable bandwidth-variable transponder, which supports sliceability, multiple bit rates, multiple modulation formats, and code rate adaptability. However, $100 \mathrm{~Gb} / \mathrm{s}$ OFDM transponders can adapt to lower bit rates. Therefore, fractional-bandwidth services may be provided with the use of the same device. This kind of technology and the use of optical integrated circuits may offer compact and cost-effective implementation. The control plane of the elastic optical network must support many unique properties, such as (i) optical channels are allowed to be flexible in size or width, (ii) optical channels can support various modulation formats, (iii) sub and super wavelength concept, (iv) support of multi-path routing of the composing waveband members of a split spectrum super-channel, (v) fast restoration upon failure, with or without resource reservation, etc. Therefore, new control protocols need to be developed or the existing protocols should be extended in order to support these unique properties of the elastic optical network.

k) 2016, [11] - A MCh granularity of $50 \mathrm{GHz}$ is sufficient to benefit from almost the full gain in SE: A media channel (Mch) is an end-to-end entity that is routed as a single FS in each WSS along its optical path and cannot be partially switched 
in an intermediate WSS. Hence, a MCh can be seen as a pipe between two nodes in the network for which we can configure the spectral width. Bit-rate variable high symbol rate signals cannot be transported over FSs of $50 \mathrm{GHz}$ granularity in a spectrally efficient manner. Stranded bandwidth is a spectrum slice that remains unused and is not wide enough to carry an optical signal, e.g., slices of $18.75 \mathrm{GHz}$ or lower would not be able to carry traffic beyond one or two WSSs. The first alternative is to design a full flexi-grid network, i.e., with a FS granularity down to $6.25 \mathrm{GHz}$, to best accommodate any net bit rate between two nodes. This approach entails a high risk of spectrum fragmentation due to incompatible FSs, which would result in underutilization of spectrum in the network, hindering us in reaching the full expected benefit of flexi-grid networks. The planning and operational challenges of such networks must not be underestimated.The second alternative is to build a flexi-grid network where FSs are still bound to an underlying grid, e.g., 25 or $50 \mathrm{GHz}$ granularity, and bit variable transponders are used to fill the available spectrum between two nodes up to the highest possible line rate. In this scenario, the distance to be bridged determines the modulation format that can be used, which, in turn, defines the SE and the line rate that can be transported in the available FS. This is operationally, and from a planning perspective, easier to manage. The conclusion is that, with current modulation formats and for the current generation of transponders, a MCh granularity of $50 \mathrm{GHz}$ is sufficient to benefit from almost the full gain in SE, which is achievable with $12.5 \mathrm{GHz}$ granularity. This conclusion is of very high interest for practical network deployment using flexi-grid WSSs.

\section{EON DEPLOYMENT POSSIBILITIES}

System providers and network operators realize they can no longer simply keep increasing a light path?s data rate while expecting the signal to fit in the standard ITU defined $50 \mathrm{GHz}$ band. However, according to Dr. Andrew Lord, head of BT's optical core and access research, said EONs should be seen as a medium to long term solution to what follows current generation DWDM based optical networks, but it is a very exciting prospect [12]. EONs depend on the availability of BV-Ts, SBVTs and BV-WXCs. Such devices have not arrived at the marketplace yet. One of the most advanced devices is Nokia's 1830 Photonic Service Switch (PSS) which implements state of art modulation formats and coding allowing to extend the DWDM network operation up to 500 Gbps [13]. Cisco's Carrier Routing System (CRS-X) offers advanced services intelligence to enable service providers to migrate their $10 \mathrm{Gbps}$ network infrastructures to $100 \mathrm{Gbps}$ and beyond [14]. The Huawei NE40E-X1/X2-M Series Universal Service Routers (USR) are high-end IP/Ethernet solutions for service providers. NE40E-X1/X2-M series supports up to 240 Gbps capacity, high-density interfaces and rich service interface types [15].

\section{CONCLusions}

In this paper we presented a review of the EON technology. It is clearly seen that it has the potential to enable data transmission at Tbps bit rates. However, it is not likely that it will arrive at the market before 2020 for at least two reasons: (i) the capacity of the current DWDM networks will not be exhausted before 2019; (ii) the major equipment providers are investing on powerful photonic switches and intelligent routers to extend the capacity of current DWDM networks beyond 500 Gbps. There is no indication when the EON critical components, BV-Ts, SBVTs and BV-WXCs, will arrive at the market.

From the above, it can be inferred that EON deployment possibilities are far away in the future and it remains as an open question if there is any condition for the interest in OPS to regain momentum.

\section{ACKNOWLEDGEMENTS}

This work was partially supported by the Catalan Government under the contract 2014SGR-1427, and by the aid granted by the Spanish Ministry of Science and Innovation under the project SUNSET (FEDER-TEC 2014-59583-C2-2-R).

\section{REFERENCES}

[1] M. Jinno, H. Takara, B. Kozicki, Y. Tsukishima, Y. Sone, and S. Matsuoka, "Spectrum-efficient and scalable elastic optical path network: architecture, benefits, and enabling technologies," IEEE Communications Magazine, vol. 47, no. 11, pp. 66-73, November 2009.

[2] M. Jinno, B. Kozicki, H. Takara, A. Watanabe, Y. Sone, T. Tanaka, and A. Hirano, "Distance-adaptive spectrum resource allocation in spectrum-sliced elastic optical path network [topics in optical communications]," IEEE Communications Magazine, vol. 48, no. 8, pp. 138-145, August 2010.

[3] I. Tomkos and D. Klonidis, "Dynamic elastic and scalable photonic infrastructures and network architectures," in 2011 13th International Conference on Transparent Optical Networks, June 2011, pp. 1-4.

[4] M. A. Klinkowski and K. Walkowiak, "On the advantages of elastic optical networks for provisioning of cloud computing traffic," IEEE Network, vol. 27, no. 6, pp. 44-51, November 2013.

[5] G. Zhang, M. D. Leenheer, A. Morea, and B. Mukherjee, "A survey on ofdm-based elastic core optical networking," IEEE Communications Surveys Tutorials, vol. 15, no. 1, pp. 65-87, First 2013.

[6] T. Tanaka, A. Hirano, and M. Jinno, "Impact of transponder architecture on the scalability of optical nodes in elastic optical networks," IEEE Communications Letters, vol. 17, no. 9, pp. 1846-1848, September 2013.

[7] V. López, B. de la Cruz, Ã. G. de Dios, O. Gerstel, N. Amaya, G. Zervas, D. Simeonidou, and J. P. Fernandez-Palacios, "Finding the target cost for sliceable bandwidth variable transponders," IEEE/OSA Journal of Optical Communications and Networking, vol. 6, no. 5, pp. 476-485, May 2014.

[8] A. Mayoral, V. Lopez, G. de Dios, and J. P. Fernandez-Palacios, "Migration steps toward flexi-grid networks," IEEE/OSA Journal of Optical Communications and Networking, vol. 6, no. 11, pp. 988-996, November 2014.

[9] E. Hugues-Salas, G. Zervas, D. Simeonidou, E. Kosmatos, T. Orphanoudakis, A. Stavdas, M. Bohn, A. Napoli, T. Rahman, F. Cugini, N. Sambo, S. Frigerio, A. D'Errico, A. Pagano, E. Riccardi, V. López, and J. P. F. P. Giménez, "Next generation optical nodes: The vision of the european research project idealist," IEEE Communications Magazine, vol. 53, no. 2, pp. 172-181, Feb 2015. 
[10] B. C. Chatterjee, N. Sarma, and E. Oki, "Routing and spectrum allocation in elastic optical networks: A tutorial," IEEE Communications Surveys Tutorials, vol. 17, no. 3, pp. 1776-1800, thirdquarter 2015.

[11] B. Clouet, J. Pedro, N. Costa, M. Kuschnerov, A. Schex, J. Slovak, D. Rafique, and A. Napoli, "Networking aspects for next-generation elastic optical interfaces," IEEE/OSA Journal of Optical Communications and Networking, vol. 8, no. 7, pp. A116-A125, July 2016.

[12] J. Walko, "Bright future for fibre optical networks," Available at http://www.newelectronics.co.uk/electronics-technology/bright-future-for-fibre-opticalnetworks/150622/, January 2017.

[13] "Nokia 1830 Photonic Service Switch (PSS)," Available at https://networks.nokia.com/products/1830-photonic-service-switch, 2017.

[14] "Cisco Carrier Routing System," Available at http://www.cisco.com/c/en/us/products/routers/carrier-routing-system/index.html, accessed on April, 12th, 2017.

[15] "NE40E-M-Series," Available at http://www.huawei.com/us/products/data-communication/routers/ne40e-m-series/index.htm, accessed on April, 12th, 2017. 